\title{
Study on water environmental dispatch scheme for complex river network in Pearl River Estuary
}

\author{
Hua Wang ${ }^{1, \mathrm{a}}$, Wei $\mathrm{Xu}^{1}$ and Xiaoming $\mathrm{Wu}^{1}$ \\ ${ }^{1}$ Department of River \& Coastal Engineering of The Pearl River Hydraulic Research Institute, 510000 Guangzhou City, China
}

\begin{abstract}
In order to promote the construction of Guangdong-Hong Kong-Macao Greater Bay Area, we should take into account the improvement of regional water environment while developing economy in Pearl River Estuary area. The water environmental dispatch scheme of river network is an important part of water environment improvement, and the key technology of dispatch is to determine the appropriate dispatch path, timing, scale and mode. The Hengqin Central River is taken as the study area in the paper. Based on the analysis of the characteristics of Hengqin Central River, the characteristics of nearby tides and water environment, this paper determines the dispatching path of "diversion from the east to the west", the time of diversion during flood tide and drainage during ebb tide, the dispatching mode of sluice control, the dispatching scale of more water exchange during spring tide and less water exchange during neap tides, and puts forward the landscape dispatching scheme and the dilution dispatching scheme. In this paper, the semiexchange period and the rate of water exchange are taken as the evaluation indexes, and the dispatching effects of different schemes are analyzed by simulating different control schemes through water quality and quantity models. The results show that the water exchange capacity of Hengqin Central River network will be greatly enhanced when the diversion and drainage amplitude increases. The research results provide reference for water quality protection and control in the Pearl River Estuary complex river network.
\end{abstract}

\section{Introduction}

The Pearl River Estuary is close to the ocean, with a large population and a developed economy. Estuary economic development ignores the regional water environment, resulting in the deterioration of the water environment in the past. And the water environment load of the river is serious and exceeds the self-purification capacity. Effective control and strict control of pollution sources is the key to harness the river's water environment. However, the control of pollution sources is a long-term task. Before the pollution sources are effectively controlled, the water flow can be accelerated, the water self-purification capacity can be improved and the water environment capacity can be increased through the scientific regulation of water conservancy projects, which can alleviate regional water environmental problems. Especially in the tidal river network area, the water quantity is abundant and the water conservancy facilities are dense, which has favorable conditions for improving the water quality and water ecology of the river ${ }^{[1-3]}$.

\section{Survey of research area}

\subsection{Characteristics of river network area}

Taking Hengqin Central River (Fig. 1) as an example, this paper studies the improvement of water exchange by the regulation of water conservancy projects in the inland river network area around the estuary. Hengqin Island is located on the coast of the South China Sea, adjacent to Hong Kong and Macao, west to Modaomen waterway, east to Shizimen waterway, is a subtropical monsoon climate, warm and humid climate, rainy and frost-free, affected by winter cold waves and typhoons in summer and autumn, the region is accompanied by gale, heavy rain phenomenon. Rainfall in this area is abundant, and the average annual rainfall is $1886 \mathrm{~mm}$.

The river network in the study area is composed of Tianmu River, North Huanshan River, South Huanshan River and interconnected drainage channels. The Central River system is controlled by the gates on both sides of the river. Under the condition of closing the gate, the water bodies in the area are relatively closed. Hengqin Central River system is a typical complex river network area around the Pearl River Estuary. The river course is staggered, the width is different, the widest part is more than $100 \mathrm{~m}$, the narrowest part is less than $10 \mathrm{~m}$, the hydrodynamic difference is great, the flow direction is changeable, and local circulation is formed. In addition, due to the influence of tides in this area, the river flow is supported by tides when the sluice is opened. Without reasonable water conservancy project dispatching scheme, the water flow in the river network is easy to 
form reciprocating flow, which is not conducive to water exchange in the Central River ${ }^{[4]}$.

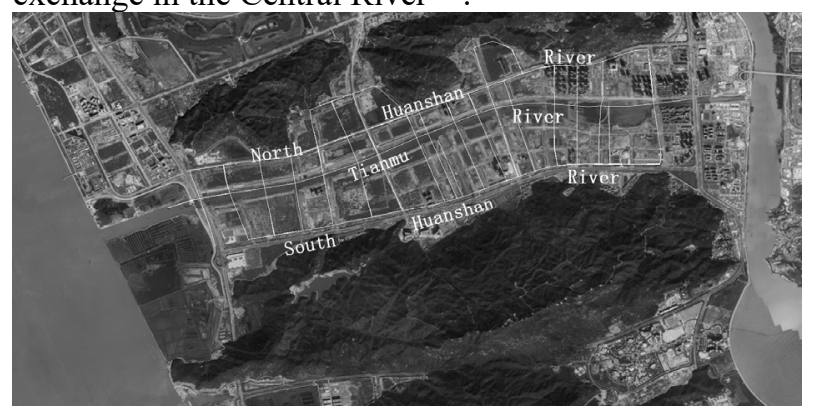

Fig 1. Outline of Hengqin Central River

\subsection{Tidal characteristics}

The tides of Hengqin Central River estuary are irregular semidiurnal mixed tides. The tides of Hengqin Central River are irregular semidiurnal mixed tides. Two high tides and two low tides occur in one diurnal cycle, lasting about 24 hours and 50 minutes. The flood tide lasts about 13 hours and 20 minutes, and the ebb tide lasts about 11 hours and 5 minutes. Tide type appears in the form of high tide - low tide - low tide - high tide, after about 12 days or 13 days, tide type becomes low tide - high tide - high tide - low tide ${ }^{[5-6]}$.

The tidal level of estuary increases from sea area to inland river, and it is higher in flood season than in dry season. When the tide rises in dry season, the high tide level of lower reaches near estuary is often higher than that of upper reaches. The tidal range reflects the tidal potential. The Pearl River estuary is a weak tidal estuary with a tidal range of about 1 meter. The average tidal range varies little in different years, and the change is very small in the year. The difference is not more than 0.20 meters $^{[7]}$.

\section{Water environmental dispatching scheme}

The key technology of water environment dispatching is to determine the appropriate dispatching path, time, scale and mode. The water quality, quantity and other related factors should be considered in the dispatching path, and the drainage direction should be determined to realize the unidirectional flow of current. The time and frequency of dispatching are determined by the tidal characteristics, the water quality of the offshore, and the water quality target of the river. Estuary area should be combined with tidal range to determine the scale of operation. The larger the tidal range is, the larger the range of drainage and drainage will be. The dispatching mode should be combined with the current situation of regional water conservancy projects and topographic characteristics to select appropriate water conservancy engineering measures, such as sluices, diversion dikes, slapping doors, pumps ${ }^{[8-11]}$.

According to the water function zoning, the upper reaches of Modaomen Waterway on the west side of Hengqin Central River are arranged with the main drinking water protection areas in Zhuhai City, and the water quality is class II. The Shizimen waterway in the East is mainly used for flood discharge and navigation, and the water quality is poor. Therefore, the "diversion from the east to the west" dispatching path has been worked out to achieve unidirectional flow of water. The water level of the outer river rises at flood tide and falls at low tide. By opening and closing the sluices on both sides of the central river, the natural tidal power is used to diversion during flood tide and drainage during ebb tide. The mean tidal range in this area is about 1 meter. We should make full use of tidal range and carry out more water exchange in the spring tide, and maintain landscape effects though less water exchange during neap tides. In the original plan, the dispatching path, time and mode are all suitable, but the scope of diversion and drainage is small and the tidal power in this area is not fully utilized. Therefore, combined with the tidal characteristics, this paper puts forward the landscape dispatching scheme and the dilution dispatching scheme.

The original plan is that during the ebb tide, the east side gate of Hengqin Central River was opened to reduce the water level of Tianmu River from 1.5 meters to 1.0 meters. During the flood tide period, the west side of Hengqin Central River sluice is opened and the east side of Hengqin Central River sluice is closed so that the water level of Hengqin Central River reaches $1.5 \mathrm{~m}$, and then the west side of the sluice closes to stop water diversion.

The landscape dispatching scheme is that during the ebb tide, the east side gate of Hengqin Central River was opened to reduce the water level of Tianmu River from 1.5 meters to 1.0 meters. During the flood tide period, the west side of Hengqin Central River sluice is opened and the east side of Hengqin Central River sluice is closed so that the water level of Hengqin Central River reaches $1.97 \mathrm{~m}$, and then the west side of the sluice closes to stop water diversion.

The dilution dispatching scheme is that during the ebb tide, the east side gate of Hengqin Central River was opened to reduce the water level of Tianmu River from 1.5 meters to 0 meters. During the flood tide period, the west side of Hengqin Central River sluice is opened and the east side of Hengqin Central River sluice is closed so that the water level of Hengqin Central River reaches $1.97 \mathrm{~m}$, and then the west side of the sluice closes to stop water diversion.

\section{Methods}

\subsection{Evaluating indicator}

How to evaluate the effect of water exchange in the process of water exchange is the main issue we are concerned about. According to the relevant research and literature results, the main evaluation factors are semiexchange period and water exchange rate ${ }^{[12]}$. The definition of semi-exchange period and water exchange rate is as follows.

The semi-exchange period reflects the water exchange capacity of different sections of the river. In this paper, the conservative material is used as a tracer to 
calculate the time needed to reduce the conservative material content by half at a certain section of the river.

Water exchange rate reflects the water exchange capacity of each section. During the process of rising tide and falling tide, the water of the river and the water of the sea constantly mix, and the water after mixing is transported to the sea. The above process is water exchange, and the formula for water exchange rate is as follows.

$$
R(r, l, t)=\left(C\left(r, l, t_{0}\right)-C(r, l, t)\right) / C\left(r, l, t_{0}\right) * 100 \%
$$

Letter $r$ refers to the river, $l$ refers to mileage, $t$ refers to time. The three variables involved in Eq. (1) are initial concentration of a channel $C\left(r, l, t_{0}\right)$, instantaneous concentration of a channel $C(r, l, t)$, instantaneous water exchange rate in a certain channel $R(r, l, t)$.

\subsection{Mathematical model and parameter setting}

The change of concentration of conservative substances in the dispatch process of the Hengqin Central River is simulated by Sobek.

One dimensional tidal current mathematical model uses the Saint-Venant equations, which are composed of continuity equation of mass conservation and momentum equation of conservation of energy. The six point implicit scheme is used in the difference scheme, and the chase method is used in numerical calculation. The basic equations are as follows.

$$
\begin{gathered}
B \frac{\partial Z}{\partial t}+\frac{\partial Q}{\partial x}=q \\
\frac{\partial Q}{\partial t}+\frac{\partial}{\partial x}\left(\beta \frac{Q^{2}}{A}\right)+g A\left(\frac{\partial Z}{\partial x}+S_{f}\right)+u_{l} q=0
\end{gathered}
$$

Variables involved in Eq. (2) and (3) are average water level of section $Z$, section flow $Q$, water area $A$, water surface width $B$, distance $x$, time $t$, side inflow $q$, momentum correction factor $\beta$, acceleration of gravity $g$, frictional slope $S_{f}$, which is calculated by Manning formula, the component of the lateral outflow velocity in the main stream direction on the unit flow $u_{l}$.

The convection diffusion equation is applied to calculate. The basic equation is as follows.

$$
\frac{\partial A C}{\partial t}+\frac{\partial Q C}{\partial x}-\frac{\partial}{\partial}\left(A D \frac{\partial C}{\partial x}\right)=-A K C+C_{2} q
$$

Variables involved in Eq. (4) are concentration of matter $C$, discrete coefficient $D$, section area $A$, linear attenuation coefficient $K$, source or sink concentration $C_{2}$, transverse inflow $q$.

According to the cross-section form of Hengqin Central River and the relevant research results of Pearl River Estuary, the roughness of the river is $0.014 \sim 0.025$ [13]. The initial water level is 1.5 meters. In order to facilitate the statistical analysis of the semi-exchange period and water exchange rate of Hengqin Central River, it is assumed that the initial concentration of pollutants in the Central River is $10 \mathrm{mg} / \mathrm{L}$ and the concentration of pollutants in the sea is $0 \mathrm{mg} / \mathrm{L}$.

\section{Results and discussion}

\subsection{Comparative analysis of semi-exchange period}

According to the simulation results, the semi-exchange period of Hengqin Central River is shown in Fig 2, and the position of channel section is shown in Fig 3. In the original plan, the semi-exchange period of the river near the West sluice is only 21.3 hours, the semi-exchange period of the North Huanshan River is about 46.5 hours, the semi-exchange period of the South Huanshan River is about 53.6 hours, and the conservative substance concentration of the water near the East sluice is still above $5 \mathrm{mg} / \mathrm{L}$ at the end of the calculation period (132 hours). In the landscape dispatching scheme, the semiexchange periods of the sections of Tianmu River ranged from 20.3 hours to 53.5 hours, those of the sections of North Huanshan River ranged from 24.2 hours to 68.7 hours, and those of South Huanshan River ranged from 47.3 hours to 72.3 hours. In the dilution dispatching scheme, the semi-exchange periods of the sections of Tianmu River ranged from 11.3 hours to 30.5 hours, those of the sections of North Huanshan River ranged from 12.7 hours to 51.8 hours, and those of South Huanshan River ranged from 21.2 hours to 74.0 hours.

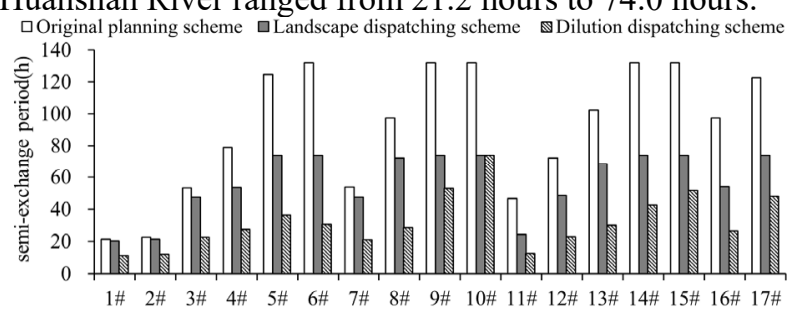

Fig 2. Semi-exchange period of Hengqin Central River for various schemes.

In the original plan, Hengqin Central River has a long water exchange period and a low water exchange efficiency. The south-eastern end of Nanhuanshan River is supported by the tidal power on both sides, and the water exchange capacity is poor, which can easily lead to pollution accumulation and deterioration of local water quality. Landscape dispatching scheme and dilution dispatching scheme both increase the diversion and drainage range and shorten the semi-exchange period of the local reach of the central river. Compared with the original planning scheme, the semi-exchange period of Hengqin Central River is shortened by 1 hour to 58 hours in landscape dispatching scheme, and the semiexchange period of Hengqin Central River is shortened by 10 hours to 102 hours in dilution dispatching scheme. According to the simulation results of landscape dispatching scheme and dilution dispatching scheme, the half-exchange period of $10 \#$ section cecum area is 74 hours, which is less than the original semi-exchange period of 132 hours. The diversion amplitude of landscape dispatching scheme and dilution dispatching scheme are the same, and the drainage amplitude is different, which indicates that the change of diversion amplitude has greater influence on the conservative substance concentration of $10 \#$ section. 


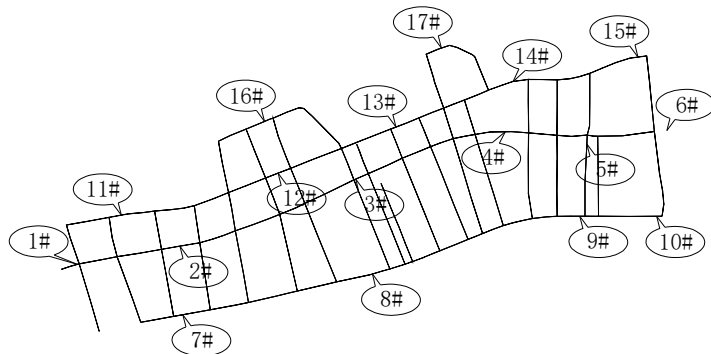

Fig 3. Schematic diagram of generalized river network and observation section.

\subsection{Comparative analysis of water exchange rate}

According to the simulation results of the model, the water exchange rate of Hengqin Central River is shown in Table 1.In the original plan scheme, the water exchange rate of Hengqin Central River is $14.55 \%$ after once dispatch, the water exchange rate of Hengqin Central River is $28.90 \%$ after twice dispatch, and the water exchange rate of Hengqin Central River is $43.41 \%$ after three times dispatch. In the landscape dispatching scheme, the water exchange rate of Hengqin Central River is $22.17 \%$ after once dispatch, the water exchange rate of Hengqin Central River is $44.10 \%$ after twice dispatch, and the water exchange rate of Hengqin Central River is $65.09 \%$ after three times dispatch. In the dilution dispatching scheme, the water exchange rate of Hengqin Central River is $48.55 \%$ after once dispatch, the water exchange rate of Hengqin Central River is $83.54 \%$ after twice dispatch, and the water exchange rate of Hengqin Central River is $94.19 \%$ after three times dispatch.

Table 1. Water exchange rate of Hengqin Central River.

\begin{tabular}{|c|c|c|c|}
\hline \multirow{2}{*}{$\begin{array}{c}\text { Water } \\
\text { exchange } \\
\text { times }\end{array}$} & $\begin{array}{c}|c| \\
\text { Original } \\
\text { planning } \\
\text { scheme }\end{array}$ & $\begin{array}{c}\text { Landscape } \\
\text { dispatching } \\
\text { scheme }\end{array}$ & $\begin{array}{c}\text { Dilution } \\
\text { dispatching } \\
\text { scheme }\end{array}$ \\
\hline Once & 14.55 & 22.17 & 48.55 \\
\hline Twice & 28.90 & 44.10 & 83.54 \\
\hline Three times & 43.41 & 65.09 & 94.19 \\
\hline
\end{tabular}

Compared with the original planning scheme, the landscape dispatching scheme and the dilution dispatching scheme improve the efficiency of water exchange. The drainage amplitude of the landscape scheme and the dilution scheme are all larger than that of the original plan. The replacement volume of Central River water increases with the increase of drainage amplitude. Therefore, the water exchange rate of dilution dispatching scheme is the highest, followed by landscape dispatching scheme, and the smallest is the original planning scheme. After once dispatch, the water exchange rate of the landscape dispatching scheme is 1.52 times that of the original scheme, while the water exchange rate of the dilution dispatching scheme is 3.34 and 2.19 times of the original scheme and the landscape dispatching scheme respectively.

\section{Conclusions}

Hengqin Central River belongs to the typical river network area in the Pearl River Estuary, where river network is dense. The water self-purification ability of the river network area is limited by the weak hydrodynamic conditions. It is of great significance to improve the water environment of Hengqin Central River by water conservancy project dispatch. Both the Modaomen and Shizimen channels on the East and west sides of Hengqin Central River are affected by the tide, so the tidal characteristics should be taken into account in hydraulic dispatching. However, the inefficiency of river network water environment dispatching is caused by insufficient consideration of tidal characteristics and unreasonable water diversion and drainage schemes in actual planning. In this paper, Hengqin Central River is taken as the study area, and the water exchange capacity of the Central River under different schemes is quantitatively evaluated by numerical simulation.

Based on the analysis of the characteristics of Hengqin Central River, the characteristics of nearby tides and water environment, this paper determines the dispatching path of "diversion from the east to the west", the time of diversion during flood tide and drainage during ebb tide, the dispatching mode of sluice control, the dispatching scale of more water exchange during spring tide and less water exchange during neap tides. In the original plan, the dispatching path, time and mode are all suitable, but the scope of diversion and drainage is small and the tidal power in this area is not fully utilized. This paper simulates the plan of Hengqin Central River, and finds that the river exchange capacity of the original scheme is insufficient. Combined with the tidal characteristics and increasing the scale of dispatching, the landscape dispatching scheme and dilution dispatching scheme are proposed. According to the simulation results, the overall water exchange capacity is improved, the half exchange period is generally shortened, and the water exchange efficiency is improved with the increase of the amplitude of dispatch. In the cecum area, the water exchange capacity is similar under the same water diversion amplitude and different water drainage amplitude, showing that the change of water diversion amplitude has greater impact on the cecum area. The research results provide a theoretical reference for water quality guarantee and operation in Pearl River Estuary.

\section{References}

1. Xing Chen, Qin Xu, Xinyue He, Guangbai Cui, Wanying Lu. Water Resources Protection 32, 77 (2016)

2. Jiatang $\mathrm{Hu}$, Shiyu Li. Acta Scientiae Circumstantiae 32, 825 (2012)

3. Jiatang $\mathrm{Hu}$, Shiyu Li. Journal of Hydraulic Engineering 43, 51 (2012)

4. Yong Lei, Xiaoming Wu. Pearl River 30, 24 (2009) 
5. Bo Su, Saltwater intrusion and saltwater control in the Modaomen Estuary (China Water \& Power Press, Beijing City, 2013)

6. Zijun Lv, Jiajia Feng, Xinyu Gao, Yunoing Wang, Runmeng Zhang, Jun Kong. Advances in Water Science 28, 908 (2017)

7. Wenlong Chen, Huazhi Zou, Yanjun Dong. Advances in Water Science 25, 713 (2014)

8. Guangbai Cui, Xing Chen, Long Xiang, Qicheng Zhang, Qin Xu. Journal of Hydraulic Engineering 48, 1429 (2017)

9. Xiuju Zhang, Kaisen Ding, Kai Yang. Water Resources and Power 2017, 31 (2017)

10. Wenxue He, Bing Zhou, Dongyun Chen,Chaqing Li,Qihua Xie. China Water \& Wastewater 28, 63 (2012)

11. Guiquan Xu, Changtai Chen, Yingzhou Tang. Water Resources Protection 2013, 80 (2013)

12. Zhenhua Chen, Changshui Xia, Fangli Qiao. Acta Oceanologica Sinica 39, 14 (2017)

13. Wenlong Chen, Yang Fang, Technical guidelines for flood control in Pearl River Delta and estuarine areas and case studies (China Water \& Power Press, Beijing City, 2014) 